\title{
DRY EYE DISEASE AMONG PREGNANT WOMEN AT A TERTIARY CARE HOSPITAL IN KATHMANDU.
}

\author{
Rizyal $A,{ }^{1}$ Shrestha $B,{ }^{2}$ Khadka $A^{1}$
}

${ }^{1}$ Department of Ophthalmology, ${ }^{2}$ Department of Obstetrics and Gynecology, Nepal Medical College Teaching Hospital, Attarkhel, Gokarneswar-8, Kathmandu, Nepal.

\begin{abstract}
Pregnancy is characterized by many significant changes in a woman's body which helps develop the fetus optimally. During pregnancy, there is marked maternal endocrine upregulation, hormonal profile modifications and interactions leading to dry eyes. Pregnancy causes most parts of a woman's body to change, and the eyes are no exception. Dry eye disease is a common pregnancy problem that usually begins towards the end of the first trimester. Since, there is a dearth of literature on dry eye disease during pregnancy in Nepal, a study was conducted to estimate the prevalence of dry eyes disease among pregnant women and to find its association with socio-demographic characteristics and their gestation period, at Nepal Medical College Teaching Hospital. A total of 84 pregnant women in different trimesters volunteered for this study. The upper and lower limits for estimated mean age were 26.67 years to 25.03 years at $95 \%$ confidence level. The prevalence of dry eyes was $89.3 \%$ by tear break up time test and $27.4 \%$ by Schirmer's test respectively. The association between gestational age and dry eyes by Schirmer's test was statistically significant.
\end{abstract}

\section{KEYWORDS}

Pregnancy, dry eye disease DED, Schirmer's test, Tear break up time (TBUT) test

\section{CORRESPONDING AUTHOR}

Dr Aparna Rizyal,

Associate Professor,

Department of Ophthalmology,

Nepal Medical College Teaching Hospital,

Attarkhel, Gokarneshwor-8, Kathmandu, Nepal.

Email: aparnarizyal@yahoo.com

Orcid ID: https://orcid.org/0000-0002-1874-2796

DOI: https://doi.org/10.3126/nmcj.v22i3.32640 


\section{INTRODUCTION}

Pregnancy is characterized by many significant changes in a woman's body which helps develop the foetus optimally. During pregnancy, there is marked maternal endocrine upregulation, hormonal profile modifications and interactions. ${ }^{1}$ These are required for appropriate anatomical and physiological adaptations needed for optimal fetal development, nourishment and smooth delivery at term. ${ }^{2}$ However, the physiological effects of these hormones often go beyond the reproductive system and affect other organs including eye and its adnexae. ${ }^{3}$ In the eye, all structures could be affected ranging from anterior segment to the posterior segment. ${ }^{4,5}$ In the anterior segment, tear film and intraocular pressure are often affected. However, while the intraocular pressure response to pregnancy is usually hypotensive, the accompanied changes in lacrimal function usually leads to dry eyes. ${ }^{6,7}$ A higher prevalence of dry eye has been reported in human and experimental studies during pregnancy. ${ }^{6,7}$

Pregnancy causes most parts of a woman's body to change, and the eyes are no exception. Dry eye disease is a common pregnancy ailment that usually begins towards the end of the first trimester. It usually continues throughout the pregnancy and possibly for a few months postpartum. In addition to dry eyes, other subtle eye changes can occur in pregnancy, causing irritation and sometimes difficulty in wearing contact lenses. Dry eyes in pregnancy is due to hormonal changes, particularly a decline in the male androgen hormone. The symptoms of dry eye are wide and varied: burning eyes, gritty sensation in the eyes, itchy eyes and light sensitivity. There is a decrease in the tear production which also leads to dry eyes. There is also change in the consistency of tears which contributes to the development of dry eyes. ${ }^{5-7}$

Dry eye disease (DED) is a multifactorial disorders of the tear film and ocular surface due to tear deficiency or excessive tear evaporation causing damage to interpalpebral ocular surface and associated with symptoms of foreign body sensation, dryness, blurring of vision, photophobia and tear film/instability. ${ }^{8}$ Globally the prevalence rate of DED is estimated to be between 7 to $37 \% .^{9,10}$ However, the prevalence is reported to be higher in Asian countries accounting for $60.0 \%$ to $73.3 \% .^{14-19}$

Numerous studies on dry eye disease have been conducted globally, majority of these studies however have been performed on the general population and post-menopausal women. Very few studies on dry eye disease during pregnancy have been reported. Skare ${ }^{6}$ and coresearchers assessed the prevalence of lacrimal dysfunction during pregnancy and compared it with non-pregnant women. Although, a higher prevalence of dry eye was reported among the pregnant, there was no difference in Schirmer's reading between the two groups. ${ }^{6}$ In animal model, Ding and coinvestigators explored the association between pregnancy and dry eye symptoms (DES) and a significantly higher DES was demonstrated in pregnant rabbits. ${ }^{7}$

A study conducted in Africa to study the tear film functions among pregnant women showed a higher prevalence of dry eye disease in pregnancy than that of non-pregnant women. ${ }^{11}$ A longitudinal study conducted in Nigeria showed that there was an increase in dry eye disease during the second and third trimesters of pregnancy. ${ }^{12} \mathrm{~A}$ review article by Pilas $\mathrm{M}^{3}$ et al also showed that there was an increased prevalence of dry eyes among pregnant women.

A prevalence of $22.8 \%$ of dry eye disease was reported from a hospital based study in Egypt. ${ }^{13}$ Likewise, a study among postmenopausal women in North India showed a high prevalence of dry eye disease accounting for $73.3 \%{ }^{14}$ The prevalence of dry eye disease among postmenopausal women ranges from $27 \%$ to $60 \%$ in studies reported from different parts of India. ${ }^{15-19}$

At present, there is a dearth of data on pregnancy-related ocular changes in Nepal. The few studies that have been conducted, are mainly on the retinal changes during pregnancy induced hypertension. ${ }^{1}$ These studies are mostly population based where the prevalence of dry eyes disease was reported between $66.0 \%$ to $69.0 \%$ in the general population, diabetic patients and postmenopausal women respectively. ${ }^{20-22}$ No study has been reported till date showing the prevalence of dry eye disease during pregnancy. Hence, a study that aimed to estimate the prevalence of dry eyes disease among pregnant women and to find its association with socio-demographic characteristics and gestational period of women during pregnancy was conducted at Nepal Medical College Teaching Hospital. Some sequelae of dry eye disease such as keratopathy could be vision threatening. Therefore, the outcome of this study is expected to create awareness on pregnancy related dry eye disease, which in turn will facilitate early recognition, and thereby leads towards prompt treatment where necessary. 


\section{MATERIALS AND METHODS}

This was a descriptive cross sectional study conducted at the Ophthalmology department of Nepal Medical College Teaching Hospital, from November 2019 to April 2020.Consecutive sampling of all pregnant women from 18 to 49 years was performed,to reduce the selection bias. Patients were referred from Gynecology and Obstetrics out patients department . Ethical approval was taken from the Institutional Review Committee of Nepal Medical College (NMC-IRC). Informed and verbal consent of each participant was obtained before the study after informing the participants of the purpose of the study and confidentiality of results. A detailed history including demographic data, as well as symptoms like ocular discomfort, burning sensation, gritty sensation, foreign body sensation and tearing were asked from all pregnant women who volunteered for this study. Patients suffering from ocular diseases like conjunctivitis, blepharitis, meibomian gland dysfunction, were excluded from the study.

Dry eye disease was confirmed by the following tests:

Tear break up time: Filter paper stained with Fluoroscein was inserted into the inferior fornix of the subject. The patient was asked to blink several times in order to distribute the fluorescein evenly. Under broad beam and blue cobalt light, the time interval (seconds) between the last blink and the appearance of first randomly distributed black spot on the cornea was noted using a stop watch.

TBUT was graded as follows: ${ }^{23}$

$>10 \mathrm{sec}=$ normal

$6-10 \mathrm{sec}=$ mild to moderate dry eye

$<5 \mathrm{sec}=$ severe dry eye

Schirmer's Test 1: The eye was mobbed dry and the folded end of a $35 \mathrm{~mm}$ long and $5 \mathrm{~mm}$ wide pre-calibrated (mm) Whitman no 41 filter paper was gently inserted into the junction between the lateral $1 / 3$ rd and medial $2 / 3$ rd of the lower fornix without touching the cornea. The extent of the wetting $(\mathrm{mm})$ after 5 minutes using stop watch was recorded as the tear film function. It was graded as follows ${ }^{24}$ :

$>15 \mathrm{~mm}$ after $5 \mathrm{~min}=$ normal

14-9mm after $5 \mathrm{~min}=$ mild dry eye

8-4mm after $5 \mathrm{~min}=$ moderate dry eye

$<4 \mathrm{~mm}$ after $5 \mathrm{~min}=$ severe dry eye

Diagnosis was made if either or both the tests were positive. Anterior segment evaluation was done using slit lamp biomicroscopy.
The obtained data was entered in Microsoft Excel and analyzed with SPSS version 16 . Chi square test was used for statistical analysis (to measure the association between dependent and independent variables). $P$ value less than 0.05 was considered significant.

\section{RESULTS}

A total of 84 women in all trimesters of pregnancy participated in this study. The minimum age was 18 years and maximum 33 years. The upper and lower limits for estimated mean age were 26.67 years to 25.03 years at $95 \%$ confidence level. Slightly more than half (59.5\%) of the participants were Janjatis and one third were Khus/Aryans (33.3\%). Dalits and Madhesis had equal participation of 3.6\%. Based on professional classification,over three quarters of the participants were homemakers accounting for $76.2 \%$. (Table 1 )

\begin{tabular}{|lcc|}
\multicolumn{4}{|c|}{ Table 1: Socio demographic } \\
characteristics of the patients
\end{tabular}

The prevalence of dry eye disease by TBUT test was $89.3 \%$ whereas the prevalence of dry eyes disease by Schirmer test was 27.5\% (Table 2).

Table 2: Frequency of dry eyes in TBUT test and Schirmer's test

\begin{tabular}{lllc|} 
Dry eyes & $\mathbf{n}$ & $\%$ & $95 \% \mathrm{CI}$ \\
TBUT test & 75 & 89.3 & $89.3 \pm 6.4$ \\
Schirmer's test & 23 & 27.4 & $27.4 \pm 3.9$ \\
\hline
\end{tabular}


Table 3: Comparison of dry eyes between TBUT test and Schirmer's test

\begin{tabular}{|lcccc|} 
& \multicolumn{2}{c}{ TBUT } & \multicolumn{2}{c|}{ Schirmer's } \\
& n & $\%$ & $\mathbf{n}$ & $\%$ \\
$\begin{array}{l}\text { Mild to moderate } \\
\text { dry eyes }\end{array}$ & 44 & 52.4 & 13 & 15.5 \\
Severe dry eyes & 31 & 36.9 & 10 & 11.9 \\
$\quad$ Total & 75 & 89.3 & 23 & 27.4 \\
\hline
\end{tabular}

\section{DISCUSSION}

Dry eye disease (DED) is a multifactorial disorder of the tear film and ocular surface due to tear deficiency or excessive tear evaporation causing damage to interpalpebral ocular surface and associated with symptoms of foreign body sensation, dryness, blurring of vision, photophobia and tear film/ instability. ${ }^{8}$ Globally the prevalence rate of DED is estimated to be between 7 to $37 \% .^{9,10}$ However, the

\section{Table 4: Association of dry eyes and gestation period in TBUT test}

\begin{tabular}{lccccc} 
Gestational age & \multicolumn{2}{c}{ Normal eyes } & \multicolumn{2}{c}{ Dry eyes } & P value ( Chi square test) \\
& $\mathbf{n}$ & $\%$ & $\mathbf{n}$ & $\%$ & \\
$\mathbf{1}^{\text {st }}$ trimester & 1 & 1.1 & 12 & 14.3 & 0.789 \\
$\mathbf{2}^{\text {nd }}$ trimester & 4 & 4.8 & 25 & 29.8 & \\
$\mathbf{3}^{\text {rd }}$ trimester & 4 & 4.8 & 38 & 45.2 & \\
Total & 9 & 10.7 & 75 & 89.3 &
\end{tabular}

\section{Table 5: Association of dry eyes and gestation period in Schirmer's test}

\begin{tabular}{lccccc} 
Gestational age & \multicolumn{2}{c}{ Normal eyes } & \multicolumn{2}{c}{ Dry eyes } & P value ( Chi square test) \\
& $\mathrm{n}$ & $\%$ & $\mathrm{n}$ & $\%$ & \\
$\mathbf{1}^{\text {st }}$ trimester & 6 & 7.1 & 4 & 4.8 & 0.048 \\
$\mathbf{2}^{\text {nd }}$ trimester & 22 & 26.2 & 9 & 10.7 & \\
$\mathbf{3}^{\text {rd }}$ trimester & 33 & 39.3 & 10 & 11.9 & \\
Total & 61 & 72.6 & 23 & 27.4 & \\
\hline
\end{tabular}

The comparison between the different grades of dry eyes in TBUT test and Schirmer's test is exhibited in Table 3.

Association between gestational period and dry eyes in TBUT test and Schirmer's test are shown in Tables 4 and 5, where the association between gestational period and dry eyes was statistically significant in Schirmer's test, ( $p$ value 0.048), but not in TBUT test ( $p$ value 0.789 ). It is observed that in both tests the frequency of dry eyes increases with the increase in the gestational period.

Though the prevalence of dry eyes among pregnant women was high in TBUT test amounting to $89.3 \%$, it was observed that more than $90 \%$ (92.9\%) had no symptoms of dry eyes like burning sensation,foreign body sensation,tearing and gritty sensation.This stresses the importance of carrying out TBUT test to assess the presence of dry eye disease during pregnancy. prevalence is reported to be higher in Asian countries ranging from $60.0 \%$ to $73.3 \% .^{14-19}$

In the global index, dry eye studies were mostly focused on the general population and postmenopausal women. However, the few studies that have been carried out on pregnancy and dry eye disease have shown an association between them. During pregnancy, all structures in the eye could be affected ranging from anterior segment to the posterior segment. ${ }^{4,5}$ In the anterior segment, tear film and intraocular pressure are often affected. However, while the intraocular pressure response to pregnancy is usually hypotensive, the accompanied changes in lacrimal function usually leads to dry eyes. ${ }^{6,7}$ A higher prevalence of dry eye has been reported in human and experimental studies during pregnancy. ${ }^{6,7}$

A total of 84 females in different trimester of pregnancy were recruited for this study, the age ranged from 18 to 49 years, the estimated 
mean age ranged from 26.67 years to 25.03 years. Slightly less than $50 \%$ of the patients were in the age group 23 to 27 years accounting for $40.5 \%$. This is in concordance to a study in Africa . ${ }^{12}$

The presence of DED among pregnant women has been reported in previous studies. ${ }^{6,7,11,12,25}$ The prevalence of DED in this study was found to be $89.3 \%$ in TBUT test and $27.4 \%$ in Schirmer's test. The high prevalence of DED found in the present study was similar to that reported in a study conducted among pregnant women by Skare ${ }^{6}$ et al, who attributed the high prevalence of DED to lacrimal dysfunction. There was higher prevalence of DED in TBUT than in Schirmer's test,which is similar to our findings.The present study is also similar to studies performed in Africa,where the prevalence of dry eyes was $75.2 \%$ in TBUT test and $24.5 \%$ in Schirmer's test respectively. ${ }^{25}$ The findings of the present study were however, inconsistent with those of Waheed A W et al ${ }^{11}$ who reported a decrease in the TBUT among pregnant women, but an increase in Schirmer's test values. This disparity might be due to the dissimilarity of study designs,as their study was based on case-control design, while ours was based on cross-sectional design.

A prevalence of $22.8 \%$ of dry eye disease by Schirmer's test was reported from a hospital based study in Egypt. ${ }^{13}$ A study in North India among postmenopausal women showed a high prevalence of dry eye disease accounting for $73.3 \% .{ }^{14}$ Both these findings are consistent with our results. Studies with results similar to ours shows the prevalence of dry eye disease among postmenopausal women ranging from $27 \%$ to $60 \%$ in studies reported from different parts of India. ${ }^{15-19}$

A study on the prevalence of dry eye diseases in Nepal conducted on the general population, reported the prevalence of dry eyes disease as $66.0 \%{ }^{20} \mathrm{~A}$ prevalence of $61 \%$ of dry eye disease among diabetics in a tertiary care hospital was reported by Singh ${ }^{21}$ and a prevalence of $69 \%$ dry eye disease among postmenopausal women in a study in eastern Nepal. ${ }^{21,22}$ The difference in the present study and these studies can be attributed to the dissimilarity in the target population.

In this study, the prevalence of DED was highest in the third trimester in both TBUT test and Schirmer's test accounting for $45.2 \%$ and $10.8 \%$ respectively. It is observed that in both tests the frequency of dry eyes increases with the increase in the gestational period. The increase in prevalence of DED in the third trimester in this study corroborated the findings reported in similar studies by Nwachukwu Nkiru Z ${ }^{12}$ et al and Wong $\mathrm{J}^{25}$ et al. Hormonal changes during pregnancy may cause a reduction in the quality and quantity of secretion from meibomian glands which will in turn enhance tear evaporation and consequently contribute to the development of DED.These hormones have been reported to increase as pregnancy progresses, reaching their peak in the third trimester. ${ }^{26}$ This might be attributed to the highest prevalence of DED and the most DED symptoms during the third trimester. A previous study in Nigeria reported an increase in estrogen and progesterone from the second to third trimester. ${ }^{28}$ In this study,the association between gestational period and dry eyes was statistically significant in Schirmer's test, ( $p$ value 0.048 ), but not in TBUT test ( $p$ value 0.789 ).

The participants in our study came to the hospital for routine antenatal visits, however only 6 patients comprising of $7.1 \%$ had clinical symptoms of DED, such as ocular discomfort, tearing, gritty sensation. More than 9 out of 10 patients (92.9\%) had no symptoms of dry eyes, however by TBUT test the prevalence of dry eye disease was $89.3 \%$. This is different to a study done by Singh $\mathrm{P}^{21}$ where the patients who complained of symptoms were $61 \%$. The discrepancy in findings can be attributed to the dissimilarity in the target population.

The meibomian gland functions normally when there is a balance between testosterone and estrogen. The mechanism behind the secretion of tear film components has been investigated for several years. For optimal functioning of meibomian glands, a delicate balance between pro secretory (testosterone) and anti-secretory hormones (estrogen) must be actively protected. This is because while testosterone enhances the development and differentiation of this gland, estrogen promotes acinar cell death leading to reduction in size of the gland and decrease in secretion. In pregnancy, though there is an upregulation in the secretion of estrogens, progesterone and testosterone, there is also a simultaneous increase in testosterone binding protein (TBP) with attendant depletion of biologically active free testosterone. ${ }^{26}$ Additionally, the sharing of the same cellular receptor by small biologically active testosterone and high level of progesterone further aggravates the reduced pro-secretory activity of testosterone on Meibomian glands.

In conclusion, this study showed that dry eye disease is a common problem during pregnancy 
which reported a high prevalence of $89.3 \%$ by TBUT test, with the highest prevalence during the third trimester accounting for $45.2 \%$. Slightly less than $50 \%$ of the patients were in the age group 23 to 27 years accounting for $40.5 \%$. More than 9 out of 10 patients (92.9\%) had no symptoms of dry eyes, even though almost $90 \%$ prevalence of dry eye disease was reported by TBUT test. Therefore, this study shows the importance of a routine eye examination to check for dry eyes disease during pregnancy. Hence, screening of all pregnant women for dry eye disease will create awareness on pregnancy related dry eye disease, which in turn will facilitate early recognition, and thereby prompt treatment where necessary.

Limitation of the study: It is possible that the volunteer bias owing to the confinement of the study within pregnant women seeking institutional delivery have affected its findings. A study encompassing women attending non -institutional (traditional) health facilities during their pregnancy seems essential to enhance the precision of the findings.

\section{REFERENCES}

1. Garg P, Aggarwal P. Ocular changes in pregnancy. Nepal J Ophthalmol 2012; 4: 150-61.

2. Omoti AE, Waziri-Erameh JM, Okeigbemen VW. A review of the changes in the ophthalmic and visual system in pregnancy. Afr J Reprod Health 2008; 12 :185-96.

3. Pilas-Pomykalska M, Czajkowskii J, Oszukowski P. Ocular changes during pregnancy. Ginekol Pol 2005; 76: 655-60.

4. Goldich Y, Cooper M, Barkana Y, et al. Ocular anterior segment changes in pregnancy. J Cat Refr Surg 2014; 40: 1868-71.

5. Akar Y, Yucel I, Akar ME, Uner M, Trak B. Longterm fluctuation of retinal sensitivity during pregnancy. Can J Ophthalmol 2005; 40: 487-9.

6. Skare TL, Gehlen ML, Silveira DMG, Uma MMDS. Pregnancy and lacrimal dysfunction. Rev Bras Ginecol Obstet 2012; 34: 170-4.

7. Ding C, Lu M, Huang J. Changes of the ocular surface and aquaporin in the lacrimal glands of rabbits during pregnancy. Mol Vis 2011; 17: 2847-55.

8. The definition and classification of dry eye disease: report of the definition and classification subcommittee of the International dry eye workshop (2007). Ocul Surf 2007; 5: 75-92.

9. Lin PY, Tsai SY, Cheng CY, Liu JH, Hsu WM. Prevalence of dry eye among elderly Chinese population in Taiwan. The Shihpat Eye Study. Ophthalmol 2003; 110: 1096-101.

10. McCarty CA, Bansal AK, Livingston PM, Stanislavsky YL, Taylor HR. The epidemiology of dry eye in Melbourne, Australia. Ophthalmol 1998; 105: 1114-9

11. Waheed AW, Ibraheem AR, Tijani AM, Oladejo S, Adepoju S. Tear Film Functions and Intraocular Pressure Changes in Pregnancy. Afr J Reprod Health 2015; 19: 118-22.

12. Nwachukwu NkiruZ, Onwubiko S, Nnemma U, AguPolycarpU, NwachukwuDanielC, Eegwuil Ifeoma R. Dry eye disease: A longitudinal study among pregnant women in Enugu, South east, Nigeria The Ocular Surface 2019; 17: 458-63.
13. Mostafa EM. Prevalence of dry eye disease in Egypt: a hospital based out-patient clinic study. $J$ Egypt Ophthalmol Soc 2016; 109: 32-40.

14. Maurya RP, Singh VP, Chaudhary S, Roy M, Srivastava T, Rajan M. Prevalence of severe dry eye disease in postmenopausal women in North India: A teaching hospital study. Indian J Obstet Gynecol Res 2019; 6: 94-6

15. Pujari MR, Kavita S, Bagare SN. Prevalence of dry eye in post-menopausal women. J Evol Med Dent Sci 2015; 4: 13005-10.

16. Majumdar M, Khandelwal R, Gangwani $\mathrm{T}$. Comparison of dry eyes in postmenopausal women with and without symptoms of dry eye.J Evol Med Dent Sci 2014; 3: 129633-38.

17. Gupta N, Prashad I, Jain R et al. Estimating the prevalence of dry eye among Indian patients attending a tertiary ophthalmology clinic. Ann Trop Med Paracitol 2010; 104: 247-55.

18. Sahai A, Malik P. Dry eyes: Prevalence and attributable risk factors in a hospital based population. Indian J Ophthalmol 2005; 53: 87- 91.

19. Basak SK, Pal PP, Basak S, Bandopadhyay A, Chaudhiri S, Sar S. Prevalence of dry eye disease in hospital based population in West Bengal: Eastern India. J India Med Assoc 2012; 110: 78994.

20. Sharma B. Dry eye: Demography and attributable risk factors. Postgrad Med J Nat'l Acad Med Sci 2011; 1: 16-22.

21. Singh P, Karmacharya S, Rizyal A. Dry eye syndrome and Type II Diabetes Mellitus. Nepal Med Coll J 2016; 18: 111-15.

22. Gurung J, Piya S and Khatri A. Prevalence of Dry Eye in Postmenopausal Women: A Study from Nepal. Ophthalmology 2019; 1: 1-5.

23. Versura P, Profazio V, Campos EC. Performance of tear osmolarity compared to previous diagnostic tests for dry eye disease. Curr Eye Res 2010; 35: 553-64.

24. American Academy of Ophthalmology. Orbit, Eyelids and Lacrimal System (Basic \& Clinical Science Course). American Academy of 
Ophthalmology $1^{\text {st }}$ edition.2002: 244-45.

25. Wong J, Ding C, Yiu S, Smith R, Goodwin T, Schechter JE. An epidemiological study of pregnancy and dry eye. Ocul Surf 2004; 3:S 127.

26. Ding C, Chang N, Fong YC, Wang Y, Trousdale MD, Mircheff AK. Interacting influences of pregnancy and corneal injury on rabbit lacrimal gland immunoarchitecture and function. Invest
Ophthalmol Vis Sci 2006; 47: 1368-75.

27. Kanova N, Bicikova M. Hyperandrogenic states in pregnancy. Physiol Res 2011; 60: 243-52.

28. Oyeyemi AO, Asaolu MF. Reproductive hormones and pregnancy-induced hypertension cases in Nigerian women. J Environ Sci Toxicol Food Technol 201; 9: 10-14. 